\title{
Seroprevalence and genotype of Toxoplasma gondii in pigs, dogs and cats from Guizhou province, Southwest China
}

\author{
Yong-Nian Li ${ }^{1^{*}}$, XinWen Nie ${ }^{1 \dagger}$, Qun-Yi Peng ${ }^{2}$, Xiao-Qiong Mu' ${ }^{1}$ Ming Zhang ${ }^{3}$, Meng-Yuan Tian² and Shao-ju Min ${ }^{4}$
}

\begin{abstract}
Background: Toxoplasma gondii is an obligate, intracellular protozoan that infects almost all warm-blooded animals, including humans, domesticated and wild animals. Recent studies of Toxoplasma gondii isolates from animals in different regions of China have shown a limited genetic diversity with the dominance of the ToxoDB PCR-RFLP genotype \#9 named as "Chinese 1". However, there is not much published information regarding its prevalence in domestic animals from Guizhou province, a subtropical region in Southwest China. The objectives of this study were to determine seroprevalence and genetic diversity of T gondii in pigs, dogs and cats in Guizhou province, Southwest China.

Findings: The anti-T. gondii lgG were detected in 70.0\%(49/70) pigs, 20.56\%(22/107) dogs and 63.16(12/19) cats. The anti-T. gondii lgM were found in $0.93 \%(1 / 107)$ dogs, $21.53 \%(4 / 19)$ cats, but not in pigs. In addition, the toxoplasma circulating antigen (CAG) were detected in 16.9\%18/70)pigs, 13.1\% (14/107) dogs and 10.5\%(2/19) cats. The T. gondii DNA were detected in 31.5\%(22/70) pigs, 3.7\%(4/107) dogs and 52.63\%(10/19) cats. Five T. gondii isolates were obtained(3 from pigs and 2 from cats). The genotype of these five isolates belonged to the predominant genotype "Chinese 1".
\end{abstract}

Conclusions: The high prevalence of T. gondii infection in pigs,cats and dogs indicated that the T. gondii infection is common in Guizhou province. Additionally, the T. gondii genotype "Chinese 1" was dominant in Southwest China.

Keywords: Toxoplasma gondii, Animal, Seroprevalence, Genotype, Guizhou province

\section{Findings}

Toxoplasma gondii is an obligate, intracellular protozoan that infects almost all warm-blooded animals, including humans, domesticated and wild animals [1,2]. These animals can serve as intermediate hosts of the parasite, harbouring tissue cysts, while cats and other felidae are the definitive hosts, shedding oocysts into the environment. Humans acquire T. gondii through the consumption of undercooked meat containing tissue cysts or through the ingestion of sporulated oocysts that can lead to life threatening disease in the foetus and immunocompromised/immunosuppressed patients e.g. transplant recipients. In general, T. gondii is an opportunistic pathogen and establishes long-lasting chronic infection. However,

\footnotetext{
* Correspondence: lyn@gmc.edu.cn

${ }^{\dagger}$ Equal contributors

'Department of Immunology, Guiyang Medical College, Guiyang 550001,

China

Full list of author information is available at the end of the article
}

T. gondii infection can cause high mortality in immunocompromised patients with HIV/AIDS.

The pathogenicity of $\mathrm{T}$. gondii is related to parasite genotypes and susceptibility of host species [3]. Based on early molecular genotyping studies, $T$. gondii isolates in North America and Europe have been classified into three genetic types (I, II, III). TypeIisolates are lethal to mice, and typeIIand III are usually less virulent for mice [4]. High genetic diversity of $\mathrm{T}$. gondii exists in Central and South America where a large number of genotypes were identified by RFLP typing [5]. To date, the three archetypical (type I, II and III) and several atypical types have been identified in China, of which the "Chinese 1" seems to be a predominant type [6].

Guizhou province is located in Yunnan-Guizhou plateau in Southwest China. Previous studies showed high seroprevalence of $\mathrm{T}$. gondii in pigs and human in Guizhou province $[7,8]$. However the data on T. gondii is still limited. Especially as, there is no epidemiological or genotype 
information on $T$. gondii in animals here. Thus, the aim of the present study was to analyse the prevalence and genetic characteristics of $T$. gondii in domestic pigs, pet dogs and stray cats in Guizhou province, southwestern China.

In the present study, animal samples (blood, heart and brain tissues) were obtained from 70 pigs, 107 dogs and 19 cats from November, 2011 to December, 2012. The blood and heart tissues of pigs were collected from Guiyang Jiawang slaughterhouse. The dog blood samples were obtained from Guiyang Dear pet clinic. The cat's blood and brain tissues were collected from stray cats, which were captured from some neighbourhoods in Guizhou province and the cats were euthanized. The anti-T. gondii IgG and IgM antibodies and the toxoplasma circulating antigens (CAG) were assayed by Toxoplasma ELISA Test Kits (Zhuhai Haitai Life Technology Company, China). DNA was extracted from heart or brain tissue $(5 \mathrm{~g})$, or blood $(1 \mathrm{ml})$ sample for PCR detection of the 529 bp repetitive DNA element of T. gondi. DNA extraction was performed using DNA extraction reagent kits (Beijing Ding changsheng biotechnology company, China). The tissue sample homogenates $(5 \mathrm{~g} / 10 \mathrm{ml})$ from seropositive animals were bioassayed in mice for isolation of the T. gondii strain, following the previously described protocol $[6,9]$. Mouse peritoneal exudates were collected and examined for viable $T$. gondii. Tissue cysts were microscopically examined as a squash preparation as described previously [10]. T. gondii tachyzoites collected from intraperitoneal fluid were cryopreserved in liquid nitrogen for long term storage. Genotyping of $T$. gondii isolates was performed using multilocus PCR-RFLP with 10 genetic markers as previously described [4]: SAG1, SAG2, SAG3, BTUB, GRA6, c22-8, c29-2, L358, PK1 and Apico. Reference strains of $T$. gondii were also used in genotyping, including type I (GT1), type II (PTG), type III (CTG) and other strains (MAS, TgCgCa1, TgCatBr5, TgWtdsc40, TgToucan(TgrRsCr1), and TgCatBr64) were kindly provided by Dr. Chunlei Su at the University of Tennessee, Knoxville USA. In addition, UPRT-1 intron sequence of T. gondii was amplified through nested-PCR. The DNA sequencing was generated by SinoGenoMax company (Beijing, China). The PCR products were digested with appropriate restriction endonucleases. The restriction fragments were run by electrophoresis. And the typing data were analyzed using ToxoDB (www.toxodb.org) database and compared with the reference strain profiles.

In this study, Toxoplasma specific IgG, IgM, CAG and 529 bp DNA fragments were tested in 196 animals including 70 pigs, 19 cats and 97 pet dogs. The anti-T. gondii IgG were detected in $70.0 \%$ (49/70) pigs, 20 . 6\% (22/107) dogs and 63.2\% (12/19) in cats. The anti-T. gondii IgM were not found in pigs, but were found in $0.9 \%(1 / 107)$ of dogs and $21.5 \%(4 / 19)$ of cats tested. In addition, the toxoplasma circulating antigen (CAG) positive rate was $16.9 \%(18 / 70)$ in pigs, $13.1 \%(14 / 107)$ in dogs and $10.5 \%(2 / 19)$ in cats. The $T$. gondii DNA were detected in $31.5 \%(22 / 70)$ pigs, $3.7 \%(4 / 107)$ dogs and $52.6 \%(10 / 19)$ cats (Table 1). Furthermore, five viable $T$. gondii isolates were obtained ( 3 from pigs and 2 from cats). These isolates displayed the identical genotype, which belongs to the Chinese 1 type. The UPRT-1 sequences from these isolates are identical and confirmed as the Chinese 1 genotype, a dominant type in China $[6,8]$ (Figure 1 and Table 2).

The present results showed high prevalence of $\mathrm{T}$. gondii (70.0\%) in pigs. It is in agreement with previous reported prevalence of $65.8 \%$ in pigs in Guizhou province [8], and $60.4 \%$ in Chongqing [11]. This high prevalence level can be explained by poor-managed facilities in this area. It was shown that if rodents and cats were controlled, as carried out on well-managed intensive farms, $\mathrm{T}$. gondii prevalence would drop drastically, in a similar way to that observed in the USA and other developed countries [12,13]. The high prevalence of $\mathrm{T}$. gondii in pigs from different farms, that were often infested with rats and cats, seems to correlate well with the high prevalence of $\mathrm{T}$. gondii in stray cats. Here, we show that $12 / 19$ (63.2\%) of stray cats were infected with $\mathrm{T}$. gondii. The prevalence of $\mathrm{T}$. gondii infection in stray cats was $63.2 \%$ in Guiyang, $57.8 \%$ in Beijing [14], $45.3 \%$ in Lanzhou [15] and $11.7 \%$ in Shanghai [16]. Whereas it was reported that $19.5 \%$ of pet dogs in Guiyang, $13.2 \%$ in Beijing [17], 11.1\% in Lanzhou [18] and $2.6 \%$ in Shanghai were infected [19]. In general, higher prevalence in cats was accompanied by higher prevalence in humans, dogs, pigs and other susceptible animals, therefore increasing the chances of environmental contamination by millions of oocysts shed by infected cats, and higher risk of ingestion of meats containing tissue cysts from infected animals [20]. Therefore, controlling

Table 1 Serological test and 529 bp detection of T. gondii in pigs, cats and dogs

\begin{tabular}{|c|c|c|c|c|c|c|c|c|c|}
\hline \multirow[t]{2}{*}{ Animals } & \multirow[t]{2}{*}{ Quantities } & \multicolumn{2}{|c|}{ lgG positive } & \multicolumn{2}{|c|}{ IgM positive } & \multicolumn{2}{|c|}{ CAG positive } & \multicolumn{2}{|c|}{ 529bpPCR positive } \\
\hline & & Samples & $\%$ & Samples & $\%$ & Samples & $\%$ & Samples & $\%$ \\
\hline Pigs & 70 & 49 & 70 & 0 & 0 & 18 & 16.88 & 22 & 31.51 \\
\hline Straying cats & 19 & 12 & 63.16 & 4 & 21.05 & 2 & 10.53 & 10 & 52.63 \\
\hline Pet dogs & 97 & 19 & 19.59 & 1 & 1.03 & 14 & 14.43 & 4 & 4.12 \\
\hline Total & 186 & 80 & 43.01 & 5 & 2.69 & 34 & 18.28 & 36 & 17.2 \\
\hline
\end{tabular}




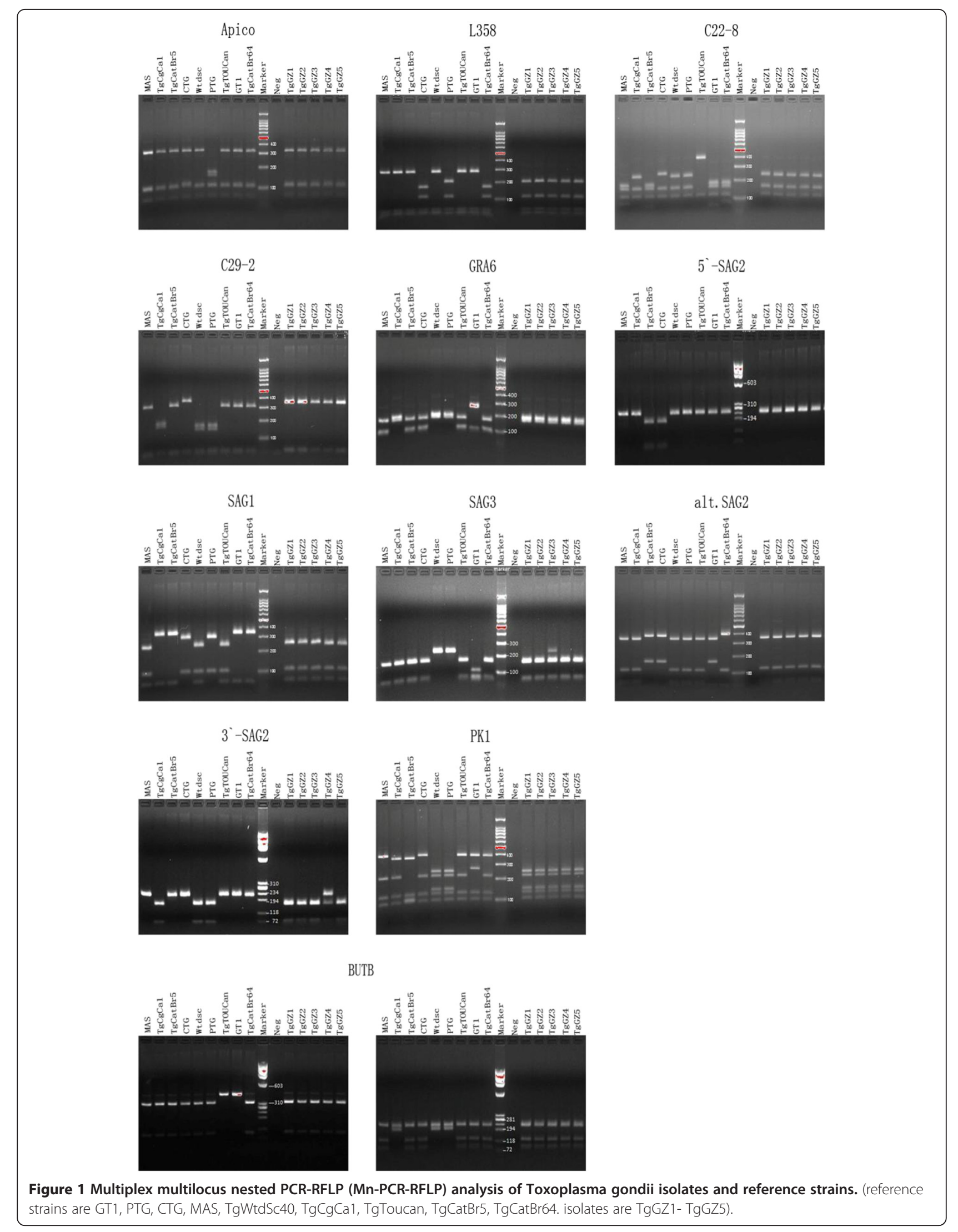


Table 2 Genotype of $T$. gondii reference strains and isolates in Guizhou province

\begin{tabular}{|c|c|c|c|c|c|c|c|c|c|c|c|c|}
\hline \multirow{2}{*}{$\begin{array}{l}\text { Reference } \\
\text { strains and } \\
\text { isolates }\end{array}$} & \multicolumn{12}{|c|}{ Markers } \\
\hline & SAG1 & $\left(5^{\prime}+3^{\prime}\right)$ SAG2 & Alt.SAG2 & SAG3 & BTUB & GRA6 & $C 22-8$ & C29-2 & L358 & PK1 & Apico & Comments \\
\hline GT1,RH88(typel) & 1 & 1 & I & I & I & I & I & 1 & I & I & I & Reference \\
\hline PTG(type II) & $\|o r\| l \mid$ & $\|$ & $\|$ & $\|$ & $\|$ & $\|$ & $\|$ & $\|$ & $\|$ & $\|$ & $\|$ & Reference \\
\hline CTG(type III) & \|lorlll & III & III & III & III & III & III & III & III & III & III & Reference \\
\hline MAS & $\mu-1$ & I & $\|$ & III & III & III & $\mu-1$ & I & I & III & I & Reference \\
\hline TgWtdSc40 & $\mu-1$ & $\|$ & $\|$ & $\|$ & $\|$ & $\|$ & $\|$ & $\|$ & I & $\|$ & I & Reference \\
\hline TgCgCa1 & I & $\|$ & $\|$ & III & $\|$ & $\|$ & $\|$ & $\mu-1$ & I & $\mu-2$ & I & Reference \\
\hline TgRsCr1 & $\mu-1$ & I & $\|$ & III & I & III & $\mu-2$ & I & I & III & I & Reference \\
\hline TgCatBr5 & । & III & III & III & III & III & । & I & I & $\mu-1$ & । & Reference \\
\hline TgCatBr64 & I & । & $\mu-1$ & III & III & III & $\mu-1$ & I & III & III & I & Reference \\
\hline TGGZ1 (pig) & $\mu-1$ & $\|$ & $\|$ & III & III & $\|$ & $\|$ & III & $\|$ & $\|$ & । & This study \\
\hline TGGZ2(pig) & $\mu-1$ & $\|$ & $\|$ & III & III & $\|$ & $\|$ & III & $\|$ & $\|$ & I & This study \\
\hline TGGZ3(pig) & $\mu-1$ & $\|$ & $\|$ & III & III & $\|$ & $\|$ & III & $\|$ & $\|$ & I & This study \\
\hline TGGZ4(cat) & $\mu-1$ & $\|$ & $\|$ & III & III & $\|$ & $\|$ & III & $\|$ & $\|$ & I & This study \\
\hline TGGZ5(cat) & $\mu-1$ & $\|$ & $\|$ & III & III & $\|$ & $\|$ & III & $\|$ & $\|$ & I & This study \\
\hline
\end{tabular}

(reference strains are GT1, PTG, CTG, MAS, TgWtdSc40, TgCgCa1, TgToucan, TgCatBr5, TgCatBr64. isolates are TgGZ1- TgGZ5).

the $\mathrm{T}$. gondii infection and contamination emission of cats is important.

There is scarce information concerning the isolation and genotyping of $T$. gondii in Guizhou province. In the present investigation, we obtained five viable $T$. gondii isolates ( 3 from pigs and 2 from cats) by bioassay in mouse. These isolates showed low virulence in mice(the data will reported in another paper). Furthermore, these isolates have identical genotype and belongs to "Chinese 1 ". Previous reports of genetic typing of $T$. gondii isolates from cats in China revealed that 15 (total 28, 88.23\%) isolates are "Chinese 1" [13]. This genotype has also been found in Guangdong province, and Hunan, and Hubei province in China. Especially in Guangdong province, 26 (total 28, 92.86\%) isolates were "Chinese 1" indicating it was the dominant genotype in that region [21]. The recent literature on genotypes revealed that 15/ 23(65.2\%) of T. gondii isolates from Anhui, and Hubei, and Guizhou province were the "Chinese 1". At the same time, typesI,II,III and other atypical types were also found in these areas [6,22]. Our results confirm that the "Chinese 1 " is a dominant isolate that is wide spread in China.

\section{Ethics statement}

All experimental animals were treated in strict accordance to the guidelines for the Laboratory Animal Use and Care from Chinese CDC and the Rules for Medical laboratory Animals (1998) from Ministry of Health, China. The protocols were approved by the Institutional Review Board (IRB) of the Institute of Biomedicine at Guiyang Medical College. All efforts were made to minimize animal suffering during the course of these studies.

\section{Competing interests}

The authors declare that they have no competing interests.

\section{Authors' contributions}

YNL conceived and designed the study, and critically revised the manuscript. EXN performed the experiments, analyzed the data and drafted the manuscript. QYP, XQM, MZ, MYT and SJM participated in the experiments. All authors have read and approved the final manuscript.

\section{Acknowledgments}

This work was supported by The National Natural Science Foundation of China (NSFC) (No.81060137). We thank the staff of Dear Animal Hospital and JiaWang Slaughterhouse who assisted in sample acquisition. We are grateful to Hong-nining and Qian-dexing and Wanglin for their advices on the experiments. We thank Dr. Chunlei Su in The Department of Microbiology, the University of Tennessee, Knoxville for technical support and constructive comments to this manuscript.

\section{Author details}

'Department of Immunology, Guiyang Medical College, Guiyang 550001, China. ${ }^{2}$ Department of Laboratory, Guiyang Medical College, Guiyang 550001, China. ${ }^{3}$ Department of Clinical Laboratory, The Fourth Hospital of Guiyang, Guiyang 550004, China. ${ }^{4}$ Department of Clinical Laboratory, Baiyun Hospital Affiliated to Guiyang Medical College, Guiyang 264000, China.

Received: 6 November 2014 Accepted: 17 March 2015

Published online: 10 April 2015

\section{References}

1. Dubey JP, Rajendran C, Ferreira LR, Martins J, Kwok OCH, Hill DE, et al. High prevalence and genotypes of Toxoplasma gondii isolated from goats, from a retail meat store, destined for human consumption in the USA. Int J Parasitol. 2011:41:827-33.

2. Hill DE, Dubey JP. Toxoplasma gondii prevalence in farm animals in the United States. Int J Parasitol. 2013:43:107-13.

3. Dubeym JP. Toxoplasmosis of Animals and Humans (Second Edition). USA: CRC Press; 2009. p. 145-59. 6

4. Su C, Zhang X, Dubey JP. Genotyping of Toxoplasma gondii by multilocus PCR-RFLP markers:A high resolution and simple method for identification of parasites. Int J Parasitol. 2006;36:1-8. 
5. Rajendran C, Su C, Dubey JP. Molecular genotyping of Toxoplasma gondii from Central and South America revealed high diversity within and between populations. Infect Genet Evol. 2012;12(2):359-68.

6. Wang L, Cheng HW, Huang KQ, Xu YH, Li YN, Du J, et al. Toxoplasma gondii prevalence in food animals and rodents in different regions of China: isolation, genotyping and mouse pathogenicity. Parasit Vectors. 2013;6:273-8.

7. Zhou P, Chen ZG, Li HL, Zheng HH, He SY, Lin RQ, et al. Toxoplasma gondii infection in humans in China. Parasit Vectors. 2011:4:165-74.

8. Hong N, Qian D, Zhang D, Hua Y, Li T, Ran L, et al. Serological investigation of pig's Toxoplasmosis in Guizhou province. Chin J Vet Parasitol. 2010;18 (4):68-70.

9. Dubey JP. Refinement of pepsin digestion method for isolation of Toxoplasma gondii from infected tissues. Vet Parasitol. 1998;74:75-7.

10. El Behairy AM, Choudhary S, Ferreira LR, Kwok OC, Hilali M, Su C, et al. Genetic characterization of viable Toxoplasma gondii isolates from stray dogs fromGiza. Egypt Vet Parasitol. 2013;193:25-9.

11. Tan Q, Li Y, Nie K, Zeng Z, Yang Z. Seroprevalent Study of Toxoplasmosis among pigs in Chongqing Area. Progress Vet Med. 2008;29(7):109-10. 115.

12. Dubey JP, Hill DE, Rozeboom DW, Rajendrana C, Choudharya S, Ferreira LR, et al. High prevalence and genotypes of Toxoplasma gondii isolated from organic pigs in northern USA. Vet Parasitol. 2012;188:14-8.

13. Dubey JP, Zhu XQ, Sundar N, Zhang H, Kwok OC, Su C. Genetic and biologic characterization of Toxoplasma gondii isolates of cats from China. Vet Parasitol. 2007;145(3-4):352-6.

14. Qian W, Wang H, Su C, Shan D, Cui X, Yang N, et al. Isolation and characterization of Toxoplasma gondii strains from stray cats revealed a single genotype in Beijing, China. Vet Parasitol. 2012;6;187(3-4):408-13.

15. Wu SM, Zhu XQ, Zhou DH, Fu BQ, Chen J, Yang JF, et al. Seroprevalence of Toxoplasma gondii infection in household and stray cats in Lanzhou, northwest China. Parasit Vectors. 2011:4:214.

16. Quan W, Wei J, YongJun C, ChunYing L, Jinlei S, Xintong L. Prevalence of Toxoplasma gondii antibodies,circulating antigens and DNA in stray cats in Shanghai, China. Parasit Vectors. 2012;5:190.

17. Yonglan Y, Lijuan F, Ming W. Serological investigation for Toxoplasmosis among Dogs and Cats in Beijing Area. Chin J Vet Med. 2006;42(5):7-9.

18. Song-Ming W, Si-Yang H, Bao-Quan F, Guang-Yuan L, Jia-Xu C, Mu-Xin C, et al. Seroprevalence of Toxoplasma gondii infection in pet dogs in Lanzhou, Northwest China. Parasites \& Vectors. 2011;4:64.

19. Haosheng $\mathrm{Q}$, Liu M, Xiangqian $\mathrm{Y}, \mathrm{Ni} \mathrm{H}$, Ye C. Toxoplasmosis prevalence among dogs in Pudong District of Shanghai. Chin J Vet Parasitol. 2007;15 (6):34-7.

20. Dubey JP, Hill DE, Jones JL, Hightower AW, Kirkland E, Roberts JM, et al. Prevalence of viable Toxoplasma gondii in beef, chicken, and pork from retail meat stores in the United States: risk assessment to consumers. J Parasitol. 2005;91(5):1082-93.

21. Yan C, Yue CL, Qiu SB, Li HL, Zhang H, Song HQ, et al. Seroprevalence of Toxoplasma gondii infection in domestic pigeons (Columba livia) in Guangdong Province of southern China. Vet Parasitol. 2011;177(3-4):371-3.

22. Zhou P, Zhang H, Lin R-Q, Zhang D-L, Song H-Q, Chunlei S, et al. Genetic characterization of Toxoplasma gondii isolates from China. Parasitol Int. 2009;58:193-5.

\section{Submit your next manuscript to BioMed Central and take full advantage of:}

- Convenient online submission

- Thorough peer review

- No space constraints or color figure charges

- Immediate publication on acceptance

- Inclusion in PubMed, CAS, Scopus and Google Scholar

- Research which is freely available for redistribution 